\title{
Low Luminosity Activity in Hickson Compact Groups
}

\author{
M. Angeles Martinez ${ }^{1}$, Ascension del Olmo ${ }^{1}$, Jaime Perea ${ }^{1}$ and Roger \\ $\mathrm{Coziol}^{2}$ \\ 1 Instituto de Astrofisica de Andalucia(IAA),CSIC, Granada (Spain) \\ geli@iaa.es, chony@iaa.es, jaime@iaa.es \\ 2 Departamento de Astronomia, Universidad de Guanajuato (Mexico) \\ rcoziol@astro.ugto.mx
}

Summary. With the aim of studying the influence of environment on the nuclear activity of galaxies, we have selected a well defined sample of 65 Compact Groups of galaxies with concordant redshift in the Hickson Catalogue [5]. In this proceeding, we present the results of the classification of nuclear activity for 42 galaxies, based on newly obtained spectral observations. In this subsample, $71 \%$ of the galaxies turned out to have emission lines in their nuclei. $73 \%$ of these emission-line galaxies were found to have characteristics consistent with low luminosity AGN (LLAGN), which makes compact groups extremely rich in such objects.

\section{Introduction}

Although environment is suspected to play an important role on the formation and evolution of galaxies, including mass assembly, star formation, morphological evolution and AGN activity, the physical details on how such connection is established are not yet well determined.

To study the connection between environment and nuclear activity (AGN or HII), systems with high galaxy density and low velocity dispersion, where the influence of gravitational encounters are optimized, are in need. In principle, these two conditions can be found in Hickson Compact Groups of galaxies (HCG). Evidence for gravitational interaction in these systems take the forms of morphological and kinematical perturbations [6] [11], the existence of tidal features such as tails or shells [10], the presence of intergalactic gas emission observable in X-rays [8] and the perturbed distribution of molecular and atomic gas [12].

For our study of nuclear activity we have selected all the groups with concordant redshift in the Hickson catalogue [5] with $\mu_{G} \leq 24.4$ (group compactness) and $z \leq 0.045$ (redshift completeness), which resulted in a statistically complete sample of 65 groups(283 galaxies). 


\section{New observations and data reduction}

New optical spectroscopic data for 42 galaxies were obtained with the CAFOS spectrograph, during a seven night run (November 2004) on the $2.2 \mathrm{~m}$ telescope of the Calar Alto Observatory (Almeria, Spain). The detector used was a $2048 \times 2048$ SITE CCD, with a plate scale of $0.53 \mathrm{arcsec} / \mathrm{pixel}$. Specifications for the two grisms used to obtain the required wavelength range can be found in Table 1 .

Table 1. Instrumental parameters

\begin{tabular}{|c|c|c|c|}
\hline Grism & Dispersion & Resolution & Spectral Range \\
\hline B100 & $2 \tilde{A} / \mathrm{px}$ & $3.74 \AA / A / \operatorname{arcsec}$ & $3200 \AA \ddot{A}-5800 \AA$ \\
\hline G100 & $2.12 \AA / \mathrm{px}$ & $3.97 \AA / \operatorname{arcsec}$ & $4900 \AA-7800 \AA$ \\
\hline
\end{tabular}

The spectra were reduced using standard procedures in IRAF and SIPL packages. After overscan and bias subtraction, flat fielding correction was performed using internal flats (taken with a quarz lamp). A correction for the different illumination along the slit was done using sky flats. Finally, cosmic-rays were removed by a median combination of different images (at least three). During each night we also obtained arc ( $\mathrm{HgCdAr}$, $\mathrm{He}$ and $\mathrm{Rb}$ ) expositions for wavelength calibration and observed three blue and three or four red spectrophotometric standard stars for flux calibration. Using our observation of standard stars, an extinction curve was determined for each night, which resulted in photometric errors less than $5 \%$ on the calibrated fluxes. The sky contribution was calculated by selecting two bands on both sides of the target galaxy. After sky subtraction we made the alignment of the spectra along the spatial axis. In figure 1 and 2 we show some examples of spectra obtained after addition of the three central spatial sections of each galaxy and after joining the spectra obtained with the two grims.

\section{Results and analysis}

The classification of activity type of the emission line galaxies was done using standard diagnostic diagrams ([1], [9]). For our classification, we use the line ratios $\log \left([\mathrm{OIII}] / H_{\beta}\right)$ and $\log \left([\mathrm{NII}] / H_{\alpha}\right)$, which are not affected by reddening and are largely insensible to errors in the photometry. In figure 3 we plot the diagnostic diagram for all the analyzed objects (the 42 spectra obtained in Calar Alto together with 68 galaxies previously classified by Coziol et al. [3][4]). In this figure we uses the empirical separation between starburst galaxies and AGNs as determined by Veilleux and Osterbrock [9], and the separation defined by Coziol [2] to distinguish between Seyfert and LINERs. 

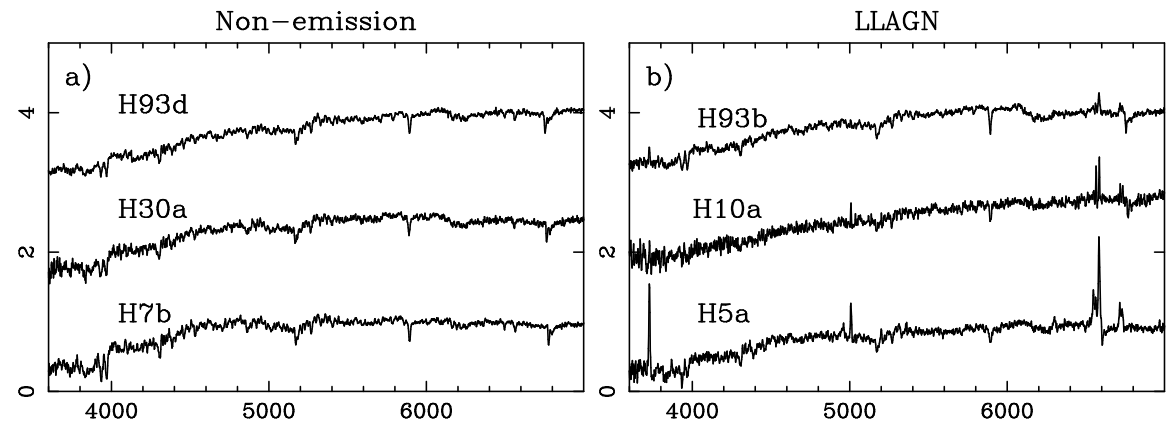

Fig. 1. Some examples of our new spectra. The flux scale is normalized to 1 and the spectra are shifted for ease of presentation.

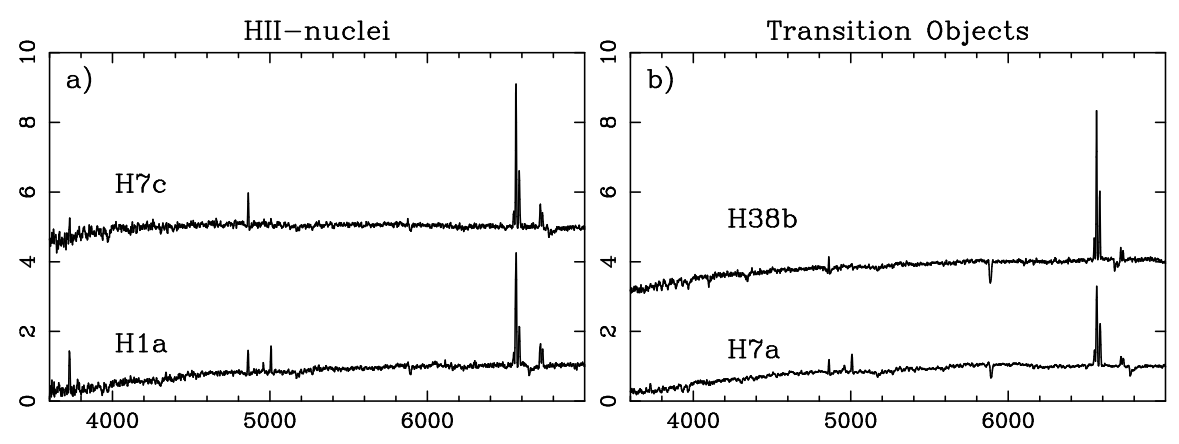

Fig. 2. Some examples of emission line galaxies.

Note that in cases where only one line ratio is available, we tentatively classify the object as AGN based on a value of $[\mathrm{NII}] / H_{\alpha}>0.6([7])$.

To summarize our classification, we have found four different types of spectra (as shown in figure 1 and 2):

- Non-emission galaxies, where only stellar absorptions features such as the CaII lines, G band, $4000 \AA$ break or Mg band are observed (Fig 1a).

- Galaxies hosting nuclear AGN activity, mainly in the form of Low Luminosity AGNs. In figure 1b, emission lines in HCG 93b are obviously diluted by intermediate age stellar population which leaves visible only the [NII] lines. In HCG 10a, on the other hand, the emission lines are more intense and the stellar contribution probably lower, but the intensity of [NII] lines are also quite strong, comparable to the intensity of $H_{\alpha}$, which also suggest an AGN nature. At it was previously shown ([3][4]) such spectral characteristics are typical of LLAGN, and usually remain even after subtracting a stellar contribution from their spectra. 
- Galaxies with star formation in their nuclei, where, as we can see in Figure $2 \mathrm{a}$, the strong emission lines are detected over a featureless continuum.

- Transition objects between HII/LINER (Fig 2b).

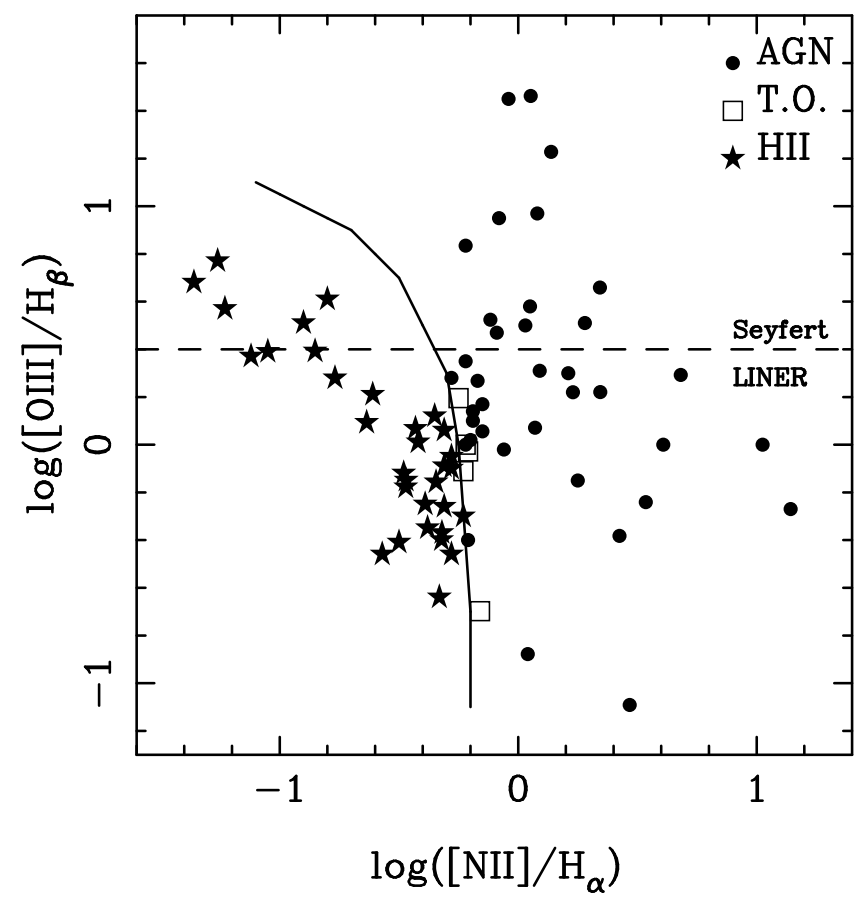

Fig. 3. Diagnostic diagram for the classified objects. The solid lines indicates the empirical separation between starburst galaxies and AGNs, as determined by Veilleux \& Osterbrock [9]. The dashed line establishes a distinction between Seyfert2 galaxies and LINERs as determined by Coziol[2].

In diagnostic diagrams, transition objects (TO; open squares in our diagnostic diagram) are located at the limit between LINER and HII-nuclear region, and are consequently thought to share features proper to both types. For instance, they show $H_{\alpha}$ lines which are more intense than the [NII] lines, although not as intense as in HII-nuclear galaxies. They also show a continuum which is not strong enough to mask emission lines. It has been suggested by Ho et al. [7] that these galaxies could be the host of an AGN (LINER or Seyfert 2) with a circumnuclear star formation region. Our present classification of these objects should be considered temporary, until a proper stellar population subtraction is performed. 
It is interesting to note that in the whole subsample classified here, we find only one galaxy, HCG 5a (in Figure 1b), with a wide component in $H_{\alpha}$ and $H_{\beta}$ characteristic of a Seyfert 1.5.

\section{Conclusions}

Of the 42 galaxies studied in this work, only 8 galaxies do not show any apparent emission. In 30 galaxies emission-lines are clearly observed, while a weak presence of [NII] or $H_{\alpha}$ is detected in four more. The percentage of emission line galaxies in our present sample amount therefore to at least $71 \%$. Within the 30 emission galaxies in our sample, 8 are classified as HII/starforming nucleus, 17 (57\%) show characteristics consistent with low luminosity AGNs with a LINER type, while the rest (5 galaxies) could represent transition like objects or TO. If TO really turn out to possess an AGN in their nucleus, that would increase the number of AGNs in our sample to $73 \%$.

If we add to our sample the 68 galaxies previously studied by Coziol et al. ([3][4]) the fraction of emission-line galaxies would amount to $67 \%$ and $60 \%$

of these would be classified as AGN (mostly LLAGN). From these results we conclude therefore that compact groups of galaxies are particularly rich in AGNs.

\section{References}

1. J.A. Baldwin, M.M Phillips \& R. Terlevich: PASP 93, 5B (1981)

2. R. Coziol: A\&A 309, 345 (1996)

3. R. Coziol, A.L.B. Ribeiro, R.R. de Carvalho \& H.V. Capelato: ApJ 493, 563 (1998)

4. R. Coziol, E. Brinks, H. Bravo-Alfaro: AJ 128, 68c (2004)

5. P. Hickson: ApJ 255, 382 (1982)

6. P. Hickson: Ann. Rev. A\&A 35, 357 (1997)

7. L.C. Ho, A.V. Fillipenko \& W.L.W. Sargent: ApJ 417, 63 (1993)

8. T.J. Ponman, P.D.J. Bourner, H. Ebeling \& H. Bhringer: MNRAS 283, 690P (1996)

9. S. Veilleux \& D.E. Osterbrock: ApJ 63, 295 (1987)

10. L. Verdes-Montenegro, A. Del Olmo, J.I. Iglesias-Paramo, J. Perea, J.M. Vilchez, M.S. Yun, \& W.K. Huchtmeier: A\&A 326, 815 (2002)

11. Verdes-Montenegro, A. Del Olmo, M.S. Yun \& J. Perea: A\&A 430, 443 (2005)

12. M.S. Yun, L. Verdes-Montenegro, A. del Olmo \& J. Perea: ApJ 475, 21 (1997) 